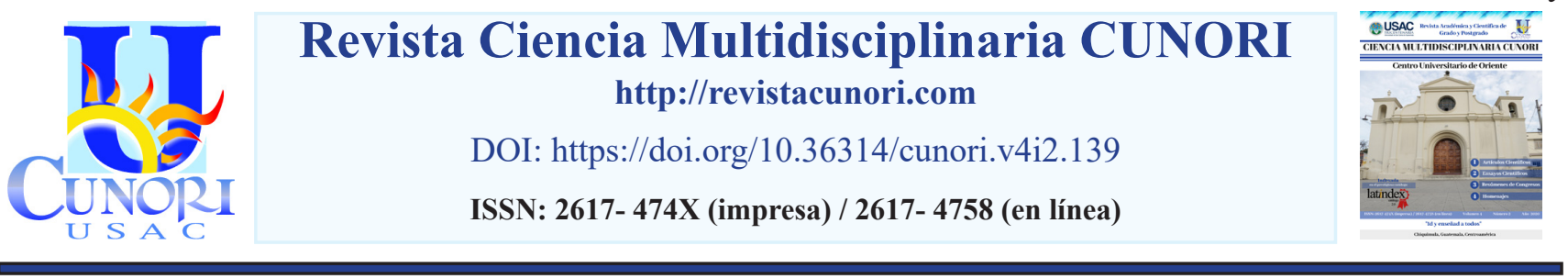

Referencia del ensayo

Alvarado-González, R. E. (2020). La utilidad de los modelos epidemiológicos para analizar la enfermedad COVID-19 en Guatemala. Revista Ciencia Multidisciplinaria Cunori, 4(2), 147-156. https://doi.org/10.36314/cunori.v4i2.139

\title{
La utilidad de los modelos epidemiológicos para analizar la enfermedad COVID-19 en Guatemala
}

\section{ArtificiThe utility of epidemiological models to analyze the diseaseCOVID-19 in Guatemala}

\author{
René Estuardo Alvarado González \\ Universidad de San Carlos de Guatemala \\ https://orcid.org/0000-0002-4444-9753 \\ Recibido: 19 de abril de 2020 / Revisado: 25 de abril de 2020 / Aceptado: 28 de junio de 2020
}

Disponible en internet el 30 de octubre de 2020

\author{
*Autor para correspondencia. \\ Resumen \\ Correo electrónico: ralvarado@cunori.edu.gt
}

— 1 interés en estudiar el comportramiento de las enfermedades infecciosas que tienden a convertirse en epidemias y ponen en Criesgo en la vida de personas, la estabilidad de las economías y los modelos de vida de las sociedades ha llevado a científicos de todo el mundo a fijar su atención en la adaptación de modelos matemáticos para estudiar el fenómeno del nuevo coronavirus SARS-Cov-2, descubierto en China a finales del año 2019 y causante de la pandemia global COVID-19, que comparte carácterísticas genéticas con su predecesor mas cercano, pero con una tasa de propagación mucho mas alta, ya que a la presenta fecha, los indicadores reportan mas de 1.6 millones de personas infectadas y mas de 100,000 muertos a nivel global. Se aborda la importancia de la modelación matemática para estimar escenarios del comportamiento de la exposición e infección de las personas a esta enfermedad, considerando el modelo epidemiológico SEIR como adaptable a la naturaleza de la pandemia COVID-19, el cual se basa principalmente en el "número reproductivo básico", para el cual se han generado estudios de referencia que permiten estimarlo para fines analíticos. Como parámetros para las ecuaciones que fundamentan el modelo, se han consultado estudios de referencia y publicaciones internacionales, con lo que se llega a demostrar la importancia de las medidas de prevención y contensión basadas en el distanciamiento social, así como la validez del modelo como herramienta útil para estimar el impacto de las estrategias a nivel de sociedad y país.

Palabras clave: modelo epidemiológico SEIR, pandemia, número reproductivo básico, SARS-Cov-2

\section{Abstract}

Tnterest in studying the behavior of infectious diseases that tend to become epidemics and put people's lives at risk, the stability of the economies and life patterns of societies has led scientists around the world to focus their attention on adapting mathematical models to study the phenomenon of the new SARS-Cov-2 coronavirus, discovered in China at the end of 2019 and causing the global pandemic COVID-19, that shares genetic characteristics with its nearest predecessor, but with a much higher rate of spread, since at the present date, the indicators report more than 1.6 million infected people and more than 100,000 deaths globally. The importance of mathematical modeling to estimate scenarios of the behavior of exposure and infection of people to this disease is addressed, considering the epidemiological model SEIR, as adaptable to the nature of the COVID-19 pandemic, which is mainly based on the " basic reproductive number", for which baseline studies have been generated to estimate it for analytical purposes. 
As parameters for the equations underlying the model, reference studies and international publications have been consulted, thus demonstrating the importance of prevention and containment measures based on social distancing, as well as the validity of the model as a useful tool to estimate the impact of strategies at the society and country level.

Keywords: SEIR epidemic model, pandemic, basic reproduction number, SARS-Cov-2

\section{Introducción}

Este estudio pretende motivar al uso de modelos epidemiológicos, basado en estadística y matemática aplicada, los cuales se han venido desarrollando y evolucionando en base a la experiencia de similares enfermedades en el pasado, donde las diversas fuentes consultadas, que incluyen publicaciones en destacados medios de divulgación en la rama de medicina, así como diversos textos de investigación universitaria, evidencian el potencial de estas herramientas no solo para predecir los niveles de propagación del virus en general, sino también como instrumento para toma de decisiones para la gestión de recursos y prevención de escenarios de calamidad, otorgando la capacidad de proyectar simulaciones con diferentes grados de precisión.

Se hará énfasis en el modelo SEIR, el cual describe en distintas fases el modelo de evolución en el proceso de contagio de una persona, desarrollado en su primera versión en 1927, el cual ha evolucionado favorablemente en su aplicación y adaptación al estudio de enfermedades en las últimas décadas, de tal manera que ha sido adaptado en otros países por sus características que le hace viable para el análisis de la actual pandemia global. Se presentan también los principales aspectos que argumentan la relevancia de la aplicación de los modelos epidemiológicos para estudiar la evolución de una enfermedad con características particulares como las que presenta COVID-19. Se analizará el principal indicador estadístico que fundamenta el modelo, el cual se conoce como el "número reproductivo básico", R0; así como otros parámetros importantes que provienen de investigaciones específicas orientadas al análisis de la trayectoria y que estiman el comportamiento de la enfermedad.

Finalmente, se analizan dos escenarios de evolución de la epidemia para el caso de Guatemala, tomando parámetros de referencia de otros países y otras adaptaciones que estiman indicadores aceptables para las características de nuestra población, demostrando que las proyecciones presentadas pueden ser útiles para apoyar a los distintos sectores de la sociedad a comprender la importancia y el impacto de la investigación científica como pilar para superar la crisis de salud que afecta en todas sus dimensiones a la sociedad postmoderna.

\section{Contenido}

\section{Antecedentes de la enfermedad covid-19}

Una epidemia, definida como una enfermedad que ataca a un gran número de individuos en un mismo lugar y durante un mismo período de tiempo, puede extenderse en una población por varios motivos, y cuando esta se extiende a varios países, se convierte en una pandemia, el cual es el caso para COVID19, que viene afectando a la humanidad a nivel global desde finales del año 2019. En la figura 1 se muestran algunas de las epidemias mas importantes de la historia, reflejando el número de muertos por 
cada una, de acuerdo a datos publicados por la Organización Mundial de la Salud y el "Centro para el control y prevención de enfermedades":

Figura No. 1 Número de muertes por las principales epidemias

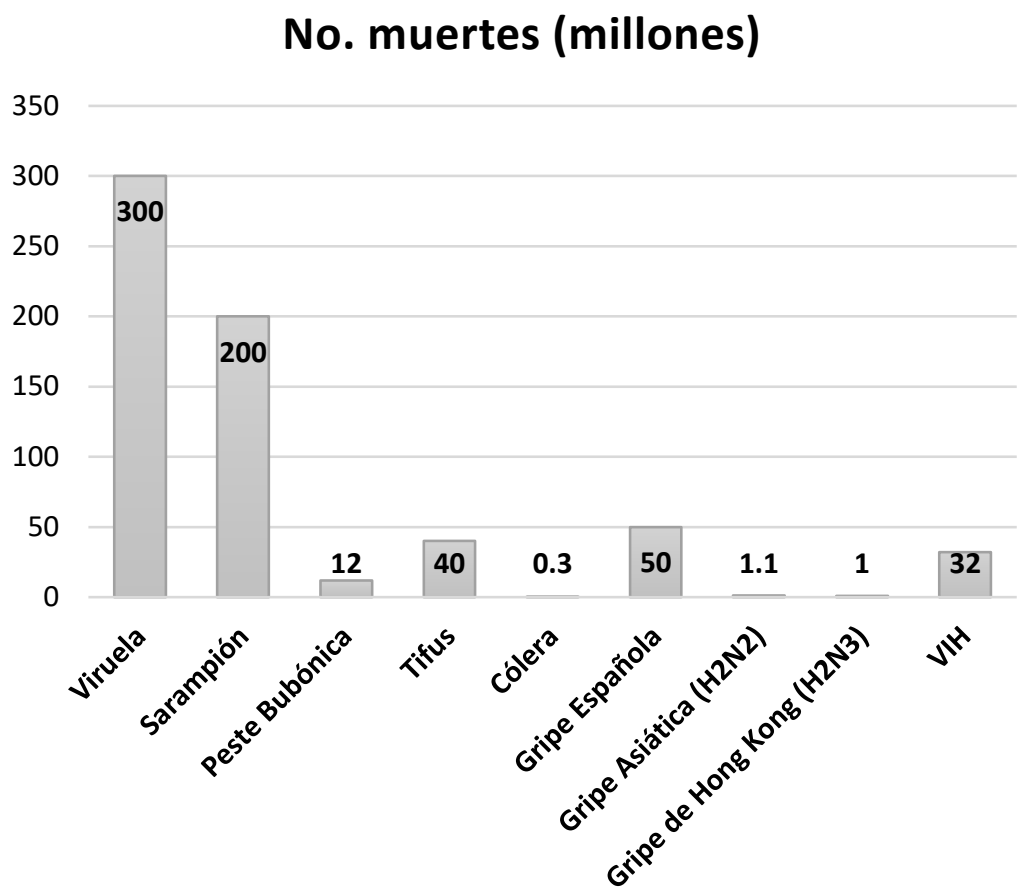

Fuente: Alvarado-González (2020). Datos publicados por la Organización Mundial de la Salud y el "Centro para el control y prevención de enfermedades":

En el año 2002, en la provincia china de Cantón, un virus hasta entonces desconocido provocó el brote de la letal enfermedad conocida como síndrome respiratorio agudo severo (SARS); donde estudios posteriores descubrieron que el patógeno causante de la enfermedad fue un coronavirus originario de un animal y transmitido a los humanos, propagándose en 29 países, infectando a mas de 8,000 personas y llevando a la muerte a unas 800 aproximadamente. Sin embargo 10 años más tarde (año 2012), surge otro letal coronavirus, conocido como MERS-Cov, causante de la enfermedad respiratoria conocida como síndrome respiratorio de medio oriente (MERS), originándose en camellos y transmitido a los humanos. Estos dos casos, desde el punto de vista de los expertos, fueron dos advertencias contundentes sobre los peligros del coronavirus.

Casi 20 años después, surge el nuevo coronavirus, conocido como SARS-Cov-2, con la característica de ser genéticamente similar en un $80 \%$ respecto a su predecesor MERS-Cov, causando a la fecha de elaboración de este artículo mas de 1.6 millones de infectados y mas de 100,000 personas muertas a nivel global. (Navas, 2020). 


\section{Los modelos matemáticos en epidemias}

Distintas teorías acerca de la naturaleza de las enfermedades infeccionas han venido siendo diseñadas por la comunidad científica, escribiéndose el primer artículo conocido de una aplicación matemática a este tipo de enfermedades a mediados del siglo XVIII, por Daniel Bernoulli, el cual contenía conocimientos matemáticos y médicos para estudiar el fenómeno de la viruela. El trabajo de Bernoulli suscitó interés y diversos investigadores continuaron su trabajo. De esta cuenta, Iranzu (2016) explica que "llegándose en 1927 a la publicación de Kermack y McKendrick, quienes escribieron acerca de un modo de predecir el tamaño final de una epidemia, planteando lo que se conoce como "teorema del umbral", el cual contenía ciertos supuestos limitantes, tal como el hecho de que la población es siempre homogénea, que llevó a generar modelos mas realistas." (p. 4). Así mismo, importante repunte de interés volvió a evidenciarse en 1990, ante el reto de entender la dinámica de enfermedades como el VIH/SIDA.

Actualmente, la aplicación de los modelos matemáticos para comprender el comportamiento de epidemias es importante, ya que pueden permitir: a) revelar relaciones de datos no siempre obvios a primera vista. b) Extraer propiedades y características de las relaciones entre elementos que de otra manera permanecerían ocultas. c) Utilizar los modelos para predecir las consecuencias de introducir cambios específicos y d) Comprender la dispersión de la enfermedad a través de una población bajo distintos escenario (Iranzu, 2016).

Las enfermedades infecciosas poseen distintos factores que vuelven imposible poder estudiarlas a todas de la misma forma, tales como, el modo de transmisión, los agentes infecciosos, la población afectada y los estados por los que puede pasar un individuo. De esta cuenta, se definen principalmente dos tipos de modelos matemáticos en epidemias, siendo los modelos estocásticos y los determinísticos. En el caso de la enfermedad COVID-19, los estudios consultados convergen en la aplicación del segundo tipo de modelo, donde por definición, la población queda determinada por un valor único y un individuo puede causar una epidemia generalizada.

Los principales modelos de este tipo, se basan en la definición de una epidemia, como una enfermedad infecciosa que prevalece únicamente un determinado tiempo o bajo unas determinadas circunstancias, considerándose fundamental el indicador R0, considerado como el número reproductivo básico (promedio estimado de personas sanas que pueden ser contagiadas por un individuo infectado), utilizado para determinar la expansión de la enfermedad (Pazos, 2020). Diversos estudios sobre el comportamiento de la enfermedad en China, realizados antes de que se adoptaran medidas drásticas de aislamiento de la población, sugieren que el valor de R0 oscila en el rango de 2.95 y 8.4, sin embargo, un estudio publicado en la revista médica británica The Lancet toma el valor del indicador en 2.68 (Wu JT, Leung K, Leung GM, 2020).

Para abordar el uso del indicador a un modelo en particular, se tomará en cuenta la recopilación de valores encontrados en 12 estudios realizados para la ciudad de Wuhan y China en general, publicados por Liu et al. en Journal of Travel Medicine, donde se refleja un valor promedio de 3.28, aproximándose 
de igual forma, con el valor supuesto de 3.4, consultado en reportes elaborados por investigadores de la Escuela de Ciencias y Físicas y Matemáticas de la USAC a finales del mes de marzo, ya que se carecen de investigaciones mas profundas en nuestro país que generen un dato significativo aplicable para un modelo epidemiológico (Chang, 2020).

\section{Características del modelo SEIR para el caso de la enfermedad COVID-19}

De acuerdo a Gutiérrez y Varona, "En matemáticas, modelizar es intentar extraer los aspectos significativamente importantes de una situación real y plasmarlos en forma de expresiones y ecuaciones matemáticas" (2020). Los modelos no pueden captar toda la realidad ni incluir todas las posibles variables involucradas, lo que hace que las epidemias no sean un caso sencillo de estudio, aunque pueden ser un instrumento de mucha utilidad para hacer variar la evolución del fenómeno epidémico al introducir medidas concretas derivado de su aplicación. El modelo SEIR se considera una herramienta viable, que aunque no irá orientada hacia una predicción real en números concretos (hacen falta datos significativos generados a partir de estudios específicos a nivel médico y social de nuestro país, que en este momento no están disponibles), sí que puede utilizarse para reflejar la influencia de las medidas de prevención y contención que se estén aplicando sobre la población durante la evolución de la epidemia.

El modelo SEIR, el cual surge a partir de una adaptación del modelo SIR para epidemias creado por Kermak y McKendrick en 1927, parte del principio de que puede ser aplicable cuando los agentes infecciosos son virus. La nueva adaptación sugiere que para una "población de tamaño fijo $\square$ en la que se ha desatado una epidemia que se propaga mediante contagio, en un tiempo $\square$ los individuos pueden estar en cuatro estados distintos" Gutiérrez y V arona (2020), donde cada uno de ellos es un paso en el proceso de contagio de una persona, siendo estos: 1) Susceptibles $S(t)$. 2) Expuestos $E(t), 3)$ Infectados I(t) y 4) Recuperados R(t).

Pazos (2020), en sus apuntes y proyecciones usando modelización matemática, nos explica "las personas Susceptibles (sanas) contraen el virus y pasan a ser Expuestas. Las personas Expuestas tienen el virus, pero aún no lo transmiten debido a que existe un tiempo de incubación. Después de ese tiempo las personas Expuestas pasan a ser Infectadas. Allí es donde se da la transmisión del virus hasta que eventualmente la persona se recupera y pasa a la categoría de Recuperada".

La clave de este modelo será, entre otros parámetros, el indicador R0, que nos permitiría saber cuantas personas pasan de una categoría a otra durante un día. El método SEIR está basado en ecuaciones diferenciales, y funciona con los tres parámetros siguientes: 1) parámetro “ $\square$ ”, el cuál será la tasa de transmisión. 2) parámetro “ $\square$ ”, el cuál es la tasa de recuperación y 3) parámetro “ $\square$ ” que será la tasa de incubación. El valor del indicador, $\mathrm{R} 0=\square / \square$ (tasa básica de reproducción), representa el número de nuevos infectados producidos por un infectado si toda la población es susceptible. Se muestran a continuación las las ecuaciones diferenciales que fundamentan el modelo: 
Figura No. 2 Ecuaciones diferenciales del modelo SEIR

$$
\begin{aligned}
\frac{d S}{d t} & =\mu(N-S)-\beta \frac{S I}{N}-\nu S \\
\frac{d E}{d t} & =\beta \frac{S I}{N}-(\mu+\sigma) E \\
\frac{d I}{d t} & =\sigma E-(\mu+\gamma) I \\
\frac{d R}{d t} & =\gamma I-\mu R+\nu S \\
N & =S+E+I+R
\end{aligned}
$$

Nesse, H. SEIR Model (s.f). Basado en el método SEIR.

De acuerdo a otros estudios realizados en China sobre el comportamiento de esta enfermedad, se puede suponer como primera aproximación el valor $\square=1 \mathrm{y} \square=0.29$, para estar de acuerdo con el valor de R0 $=3.28$, indicado anteriormente. El período de incubación de la enfermedad, como lo indica el reporte de la Organización Mundial de la Salud "es el tiempo que transcurre entre la infección por el virus y la aparición de los síntomas de la enfermedad. La mayoría de las estimaciones respecto al periodo de incubación de la COVID-19 oscilan entre 1 y 14 días, y en general se sitúan en torno a cinco días”.

Por esta razón, el parámetro $\square$ del modelo se situará con un valor de 0.2 aproximadamente. De acuerdo a la descripción de Enrique Pazos, en este modelo se destaca la importancia del indicador R0, ya que si este es mayor que 1, la epidemia crece, pero si es menor que 1, la epidemia se extingue, razón por la que el objetivo de la cuarentena es bajar todo lo posible el valor de R0. (Pazos, 2020).

Suponiendo una población de 1,000,000 de personas (ciudad capital de Guatemala), en la que hay un infectado al inicio, la evolución de la epidemia de acuerdo al modelo SEIR durante 120 días se muestra a continuación: 
Figura No. 3 Evolución de la epidemia COVID-19 según modelo SEIR, escenario 1

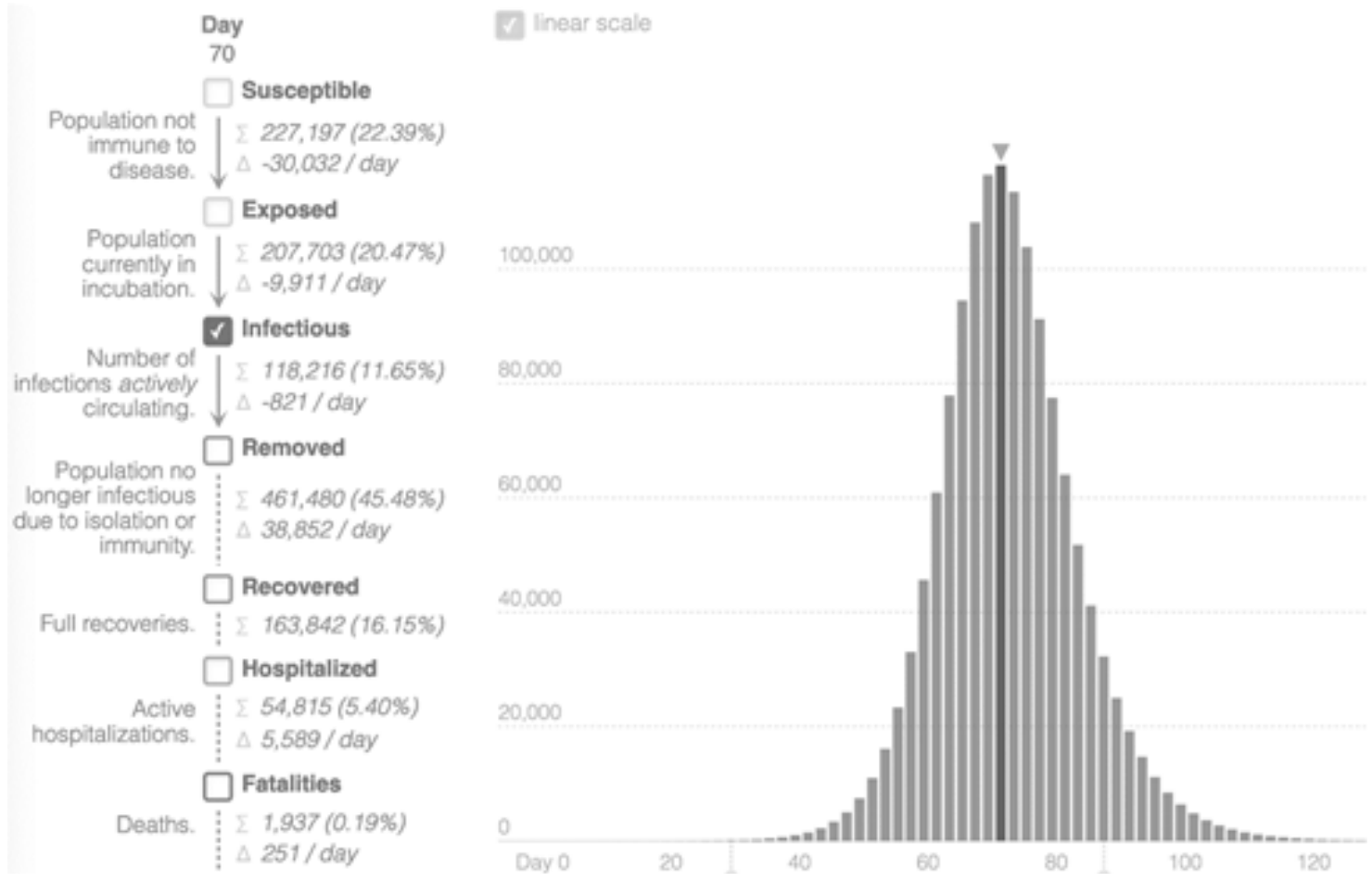

Fuente: Alvarado-González (2020). Basado en la evolución de la epidemia de acuerdo al modelo SEIR.

De acuerdo a la información reflejada en el modelo en la figura 3, aproximadamente en el día 70 (tercera semana del mes de Mayo, tomando como punto de partida el día 13-Marzo, cuando se reportó el primer contagio en Guatemala) se esperaría alcanzar el pico de personas expuestas, que oscilaría alrededor de las 200,000 personas, de las cuales aproximadamente 118,000 podrían ser casos confirmados de pacientes con COVID-19.

Las medidas de mitigación que se están tomando resultan muy importantes ya que tienen el efecto de reducir el indicador R0, lo cual disminuirá tanto el número de casos como la velocidad de propagación del virus, causando que el punto mas alto de la gráfica de personas infectadas (pico de infección) se presente mas tarde. De esta cuenta, si con las medidas de cuarentena domiciliar, el número reproductivo básico R0 disminuye a un valor de 2.1, tendríamos la siguiente gráfica, reflejada en la figura 4 , asumiendo la misma información utilizada en el escenario previo: 
Figura No. 4 Evolución de la epidemia COVID-19 según modelo SEIR, escenario 2 (aplicando cuarentena y otras medidas de contención).

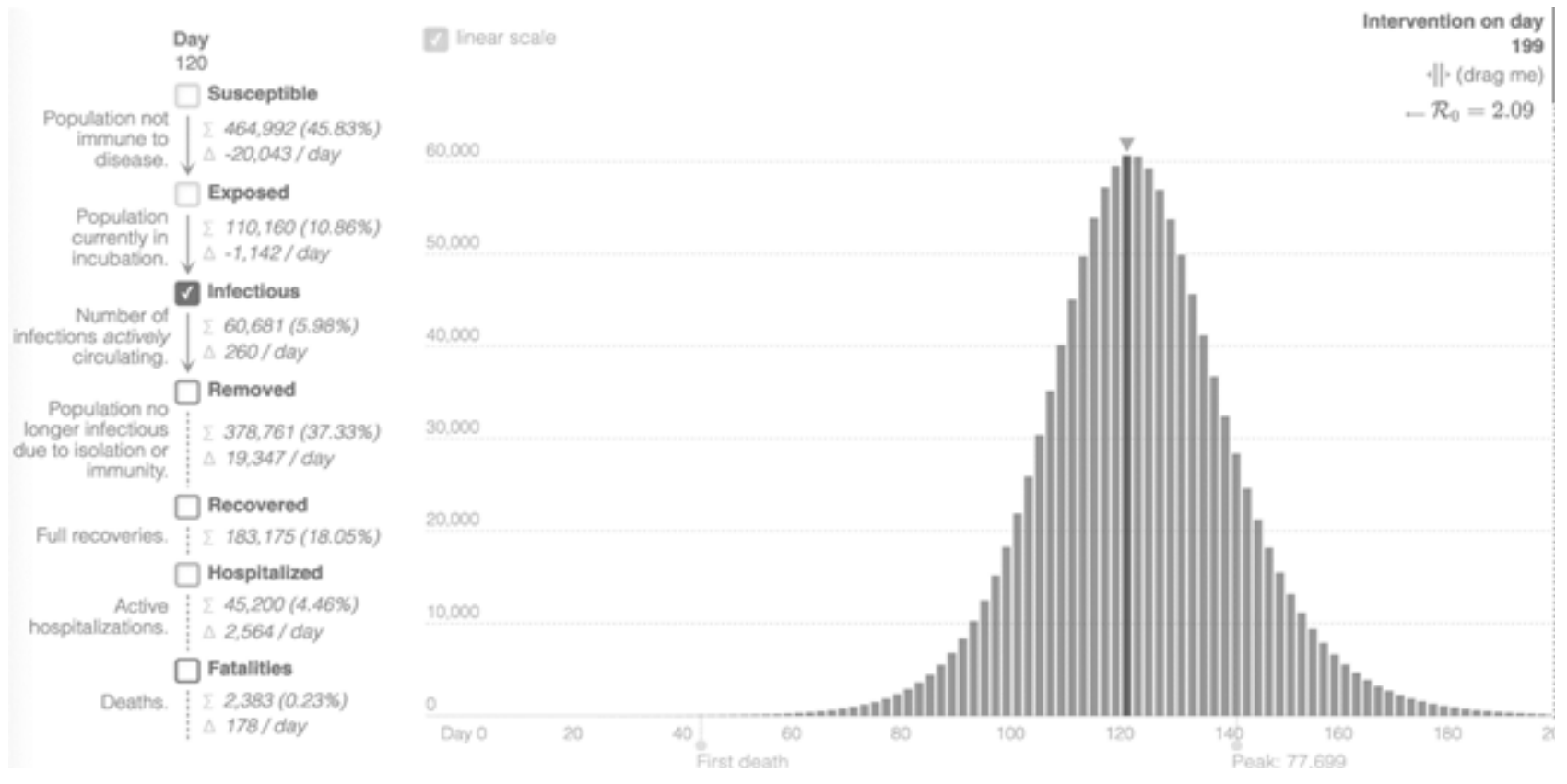

Fuente: Alvarado-González (2020). Basado en la evolución de la epidemia de acuerdo al modelo SEIR.

La figura 4 refleja la importancia de la reducción del indicador R0, ya que aproximadamente en el día 120 (4 meses después de la aparición del primer caso infectado con el nuevo coronavirus), se alcanzaría un nivel que oscila alrededor de las 60,000 personas infectadas con la enfermedad COVID-19, reflejándose una diferencia que se aproxima al doble de personas infectadas con respecto al escenario inicial. Para este análisis se ha utilizado el Epidemic Calculator, que puede encontrarse en: http://gabgoh. github.io/COVID/index.html, creado por Gabriel Goh, científico e investigador del proyecto @openai. Otra herramienta muy útil para analizar esta pandemia global es el simulador del modelo SEIR diseñado por el PhD. Hans Nesse, disponible en el sitio Web de la Universidad de Arizona, EE.UU. en http:// www.public.asu.edu/ hnesse/classes/seir.html.

\section{Conclusión}

Los antecedentes del nuevo coronavirus SARS-Cov-2, con similar genética al de su antecesor de hace 20 años, debieron haber alertado a la humanidad y propiciado la continuidad en cuanto a su investigación para fundamentar mejor a la comunidad científica para generar tratamientos contra modalidades de virus cercanamente relacionados, evitando así, un colapso a nivel de salud y un golpe contra la economía global, como el que se evidencia actualmente debido a la pandemia global de COVID-19. El modelo epidemiológico puede ser útil para estudiar un proceso y servir como instrumento importante para tomar decisiones y analizar los efectos medidas de prevención concretas, ya que permite al científico e investigador, generar distintos escenarios y estar mejor preparados al conocer datos significativos que expliquen el fenómeno de comportamiento del virus en los distintos países. 
En Guatemala, es muy grande la necesidad de fortalecer a la comunidad científica para generar estudios profundos que permitan conocer datos útiles para aplicar modelos epidemiológicos, los cuales permitirían combatir mas eficientemente al COVID-19, donde para llegar a ellos es fundamental realizar mas prueba para conocer el estado real de los habitantes con respecto a su reacción y evolución ante el nuevo coronavirus. El modelo SEIR refleja como las aglomeraciones y altas densidades de personas causan que el número reproductivo básico (R0) tenga un valor más alto, mientras que la cuarentena y el aislamiento entre individuos administrado eficazmente tienen el efecto de reducirlo, lo cual pone de manifiesto la utilidad del modelo para evidenciar ante la población el impacto de las decisiones y estrategias implementadas.

\section{Referencias}

Chang, J. y Ponciano, J. (2020). Estimación de la evolución de la epidemia COVID-19. Escuela de Ciencias Físicas y Matemáticas, Universidad de San Carlos de Guatemala. Recuperado de https://cutt. ly/NtBFPW3.

Iranzu, G. (2016). Modelos epidemiológicos basados en ecuaciones diferenciales. Facultad de Ciencia y Tecnología de la Universidad de La Rioja, España. Recuperado de https://cutt.ly/3tBFEiG.

Liu et al. (2020). The reproductive number of COVID-19 is higher compared to SARS conoravirus. Journal of Travel Medicine. doi: https://doi.org/10.1093/jtm/taaa021

Navas, E. (2020). Coronavirus: cómo el mundo desaprovechó la oportunidad de tener una vacuna lista para hacer frente a la pandemia. BBC Mundo News. Recuperado de https://cutt.ly/TtBFLe9.

Nesse, H. (2010). Global Health SEIR Model, Arizona State University. Recuperado de https://cutt.ly/ atBD26c.

Organización Mundial de la Salud (2020), Sitio Web Mundial. Recuperado de https://cutt.ly/FtBDHmG.

Pazos, E. (2020). Matemática del distanciamiento social, un hilo para incrédulos. Agencia Ocote, Guatemala. Recuperado de https://cutt.ly/TtBGe6w.

Pazos, E. (2020). Apuntes sobre modelos matemáticos para COVID-19 y proyecciones usando el calculador de epidemias online. Materia Viva. Recuperado de https://cutt.ly/KtBFdAY.

Wu JT, Leung K, Leung GM. Nowcasting and forecasting the potential domestic and international spread of the 2019-nCoV outbreak originating in Wuhan, China: a modelling study, The Lancet, 2020. Recuperado de https://cutt.ly/ItMVtx3. doi: https://doi.org/10.1016/S0140-6736(20)30260-9

\section{Sobre el autor}

\section{René Estuardo Alvarado González}

Magister en Administración de Empresas por la Universidad Rafael Landívar se especializó en Educación Virtual para el Nivel Superior es Ingeniero en Ciencias y Sistemas por la Universidad de San Carlos de Guatemala. Trabaja como Profesor titular universitario, colabora como asesor de proyectos de investigación y extensión universitaria, experiencia educativa y profesional en creación y aplicación de tecnologías de la información y comunicación. 
Copyright (c) René Estuardo Alvarado González

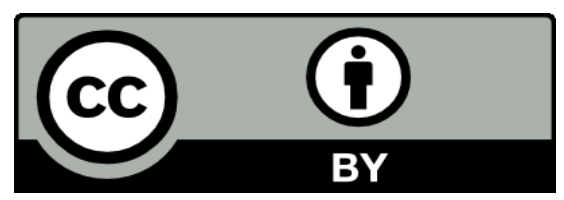

Este texto está protegido por una licencia CreativeCommons 4.0.

Usted es libre para compartir, copiar y redistribuir el material en cualquier medio o formato y adaptar el documento, remezclar, transformar y crear a partir del material para cualquier propósito, incluso comercialmente, siempre que cumpla la condición de atribución: usted debe reconocer el crédito de una obra de manera adecuada, proporcionar un enlace a la licencia, e indicar si se han realizado cambios. Puede hacerlo en cualquier forma razonable, pero no de forma tal que sugiera que tiene el apoyo del licenciante o lo recibe por el uso que hace. 